\title{
Skaleneffekte im Gesundheitswesen
}

Richard O. Binswanger

Korrespondenz:

Dr. med. Richard O. Binswanger Radiologie und

Nuklearmedizin FMH

Führungsschule Bodensee

Münsterlingen

Oberer Seeweg 9

CH-8597 Landschlacht

r.binswanger@bluewin.ch

fsb-spital.ch

\begin{abstract}
Die Industrialisierung des Gesundheitswesens
Die Industrialisierung des Gesundheitswesens schreitet voran. Merkmale sind unter anderen das Aufkommen von Spital- oder Laborketten sowie Zusammenschlüsse von Privatpraxen und Spitälern. Auch internationale Grossanbieter drängen in die Schweiz. Ein Treiber dieser Entwicklung ist die Vorstellung, im Gesundheitswesen seien Skaleneffekte (Economies of Scale) gleich wirksam wie in der Industrie oder im Bankenwesen.
\end{abstract}

\section{Was sind Skaleneffekte?}

Dies sind Kostenvorteile, die ein Unternehmen durch Expansion erlangt [1]. Diese können dringend notwendig sein. So hält Fiat-Chef Marchionne für einen Automobilkonzern die Produktion von 6 Millionen Autos pro Jahr für notwendig, um überleben zu können [2].

\section{Positive Skaleneffekte}

Peter S. Hanson untersuchte Trends in der privatärztlichen Radiologie in den USA [3]. Zwischen1990 und 2007 ging die Zahl der Praxen mit 1 bis 4 Ärzten von 29 auf 22 Prozent zurück. Praxen mit über 30 Radiologen nahmen von 5 auf 19 Prozent zu. Seit dem Jahr 2000 expandieren Praxen mit über 60 Fachärzten am stärksten. Als positiv erweisen sich die erleichterte Subspezialisierung, der verbesserte Zugang zu Kapital sowie grössere Ressourcen im Management und im Marketing. Dabei spielt auch die Schaffung einer Marke (Branding) eine grosse Rolle. Zudem kommt es zu einer Zunahme der Verhandlungsmacht, zum Beispiel gegenüber Kostenträgern und Lieferanten. Auch wird häufig eine Monopolstellung gesucht.

\section{Neutrale Skaleneffekte}

Die klinische Arbeit am einzelnen Patienten lässt sich nicht rationalisieren. Darum wird immer so viel Energie auf die Optimierung der Abläufe verwendet! Also kann für die Kernaufgabe der Medizin vom Grössenwachstum kein positiver Skaleneffekt erwartet werden. Er ist vielmehr neutral. Dies ist wichtig und muss von ärztlicher Seite stets wieder betont und wiederholt werden.

\section{Negative Skaleneffekte}

Die Komplexität von Unternehmen, auch im Gesundheitswesen, nimmt mit dem Grössenwachstum zu. Dies gilt vor allem für die Steuerung bzw. das Management. «The greater time required for management is often undervalued ...» [3]. Dabei steigt vor allem der

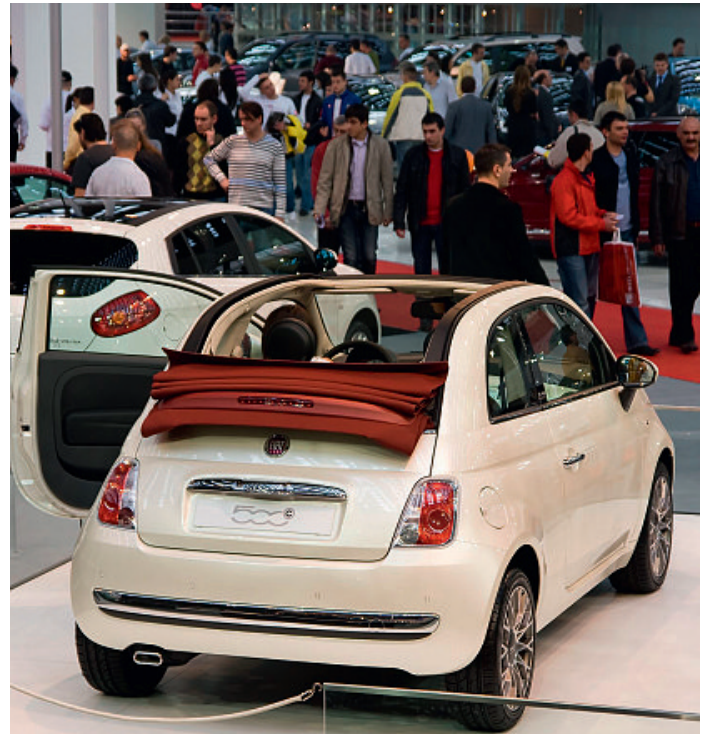

Mehr als 6 Millionen Autos zu produzieren, das sei ein positiver Skaleneffekt - sagt der Fiat-Chef. Aber was ist ein positiver Skaleneffekt im Gesundheitswesen?

zeitliche Aufwand für die interne Kommunikation und die Entscheidungsfindung, und zwar überproportional. Andere negative Effekte der Grösse sind der Verlust an Identifikation der Mitarbeiter, eine geringere Leistungs- und Opferbereitschaft sowie eine Zunahme der Risikoaversion. Ärzte, die sich in der Führung engagieren, sehen sich zunehmender Kritik ausgesetzt. Es erschallt der Ruf nach noch mehr Professionalisierung des Managements (Wenn es so weit ist, sind dann vielfach doch alle enttäuscht).

\section{Die Frage nach dem Ziel}

Die Frage nach Grössenwachstum und nach Zusammenschluss mit anderen stellt sich heute jedem Unternehmen im Gesundheitswesen. Manchmal zwingen auch äussere Entwicklungen dazu. Zunehmende Grösse bringt immer Skaleneffekte, diese können positiv, neutral oder negativ sein. Darum müssen die Ziele einer Expansion oder Fusion mit der grösstmöglichen Gründlichkeit untersucht und definiert werden, auch in Bezug auf die erhofften Skaleneffekte. Den negativen soll dabei die gebührende Beachtung geschenkt werden.

\section{Literatur}

1 http://en.wikipedia.org/wiki/Economy_of_scale

2 Tagesspiegel vom 11.6.2009.

3 Hanson PS. Private Practice Trends in US Radiology. Imaging Management. 2009;9(1):16-21. 